\title{
Antarctic snow accumulation mapped using polarization of 4.3-cm wavelength microwave emission
}

\author{
Robert J. Arthern, ${ }^{1}$ Dale P. Winebrenner, ${ }^{2}$ and David G. Vaughan ${ }^{1}$ \\ Received 7 December 2004; revised 26 October 2005; accepted 1 December 2005; published 29 March 2006.
}

[1] Different parts of Antarctica receive different amounts of snowfall each year. In this paper we map the variations of the mean annual snow accumulation across the ice sheet. We also quantify the uncertainty in our estimates more objectively than has been possible for earlier maps. The new map is produced using observations from satellites and ground-based measurements. After a logarithmic transformation, these are combined using the geostatistical method of continuous-part universal kriging to give an estimate of the snow accumulation within each cell of a rectangular grid covering Antarctica.

We also derive spatial averages over the major drainage systems of the ice sheet, along with their confidence intervals. We obtain a value of $143 \pm 4 \mathrm{~kg} \mathrm{~m}^{-2} \mathrm{a}^{-1}$ for the average rate of snow accumulation upon the grounded ice sheet of Antarctica.

Citation: Arthern, R. J., D. P. Winebrenner, and D. G. Vaughan (2006), Antarctic snow accumulation mapped using polarization of 4.3-cm wavelength microwave emission, J. Geophys. Res., 111, D06107, doi:10.1029/2004JD005667.

\section{Introduction}

[2] In this paper we seek to map the rate at which snow accumulates on the Antarctic ice sheet and also to quantify the accuracy of our map as objectively as possible. Better maps of snow accumulation are needed to understand and predict ongoing changes in the size of the Antarctic ice sheet [Wingham et al., 1998; Rignot and Thomas, 2002]. These changes are important in driving global sea level [Church et al., 2001]. A better understanding of errors is needed to optimize predictions of the future behavior of the ice sheet using data assimilation methods analogous to those used for weather prediction [Arthern and Hindmarsh, 2003].

[3] In a given year an ice sheet may grow or shrink. Growth occurs when the accumulation of snow outweighs the loss of ice from the margins. The three most important measurements for understanding and predicting changes in the size of the ice sheet are (1) the accumulation of snow upon the ice-sheet, (2) the ice-export from the margins by icebergs and melt, and (3) mass changes derived from repeated surveys of thickness or gravity. Mass changes and ice-export have been estimated for many regions of Antarctica, accurate to about $10 \%$ of the ice-flux [Wingham et al., 1998; Rignot and Thomas, 2002; Joughin and Tulaczyk, 2002]. The snow accumulation, and its likely error, are not known so precisely. This limits our knowledge of the mass changes occurring in Antarctica, and our ability to predict them [Rignot and Thomas, 2002; Vaughan et al., 1999]. For that reason, we concentrate here upon estimating the accumulation rate, together with its uncertainty.

\footnotetext{
${ }^{1}$ British Antarctic Survey, Cambridge, UK.

${ }^{2}$ Applied Physics Laboratory, University of Washington, Seattle, Washington, USA.

Copyright 2006 by the American Geophysical Union. 0148-0227/06/2004JD005667
}

[4] Recent maps of accumulation rate have been derived from compilations of data collected in situ from snowpits, snow-stakes, or ice cores, sometimes augmented by satellite observations [Vaughan et al., 1999; Giovinetto and Zwally, 2000; Huybrechts et al., 2000]. Accumulation rates are essentially equivalent to the meteorological "surface mass balance," i.e., the net mass flux from atmosphere to ice, and they have also been derived from numerical atmospheric models [Genthon and Krinner, 2001; van Lipzig et al., 2002]. The accuracy of all of these maps has usually been assessed simply by comparing one map with another.

[5] A problem occurs when the data or methods used to produce the maps are shared to some degree, since the maps may then share common errors that are not revealed by differencing. Recent surveys by Vaughan et al. [1999], Giovinetto and Zwally [2000], and Huybrechts et al. [2000] have much in situ data in common, so comparing these maps reveals little about their errors. Numerical models may also share biases [Genthon and Krinner, 2001]. Comparison of model output with compilations of the in situ data reveals discrepancies greater than $50 \%$ in places [Genthon and Krinner, 2001].

[6] In short, errors in accumulation maps are poorly constrained and may be as large as $50 \%$ in places. The distance over which errors covary is equally uncertain. Consequently, when the maps are averaged spatially over some region of the ice sheet, the likely error cannot be quantified with any great precision. Uncertainty over the precision of current maps limits their use for forecasting how Antarctica will contribute to global sea level during the coming century. Here, we seek to combine in situ data with satellite observations in a geostatistical framework that provides a more objective assessment of uncertainties.

[7] At present there is no direct way of monitoring the arrival of snow at any site on the ice sheet from space, but satellites can provide spatial information helpful to the interpolation of in situ data. Thermal microwaves are 
emitted from the uppermost tens of meters of the snowcover (in which we include "firn," older yet unconsolidated snow). As such they contain information about its properties [Zwally and Giovinetto, 1995]. Vaughan et al. [1999] report on the interpolation of an extensive compilation of in situ observations. They used the sensitivity of microwave emission to accumulation rate (following Zwally and Giovinetto [1995]) to guide this interpolation in regions where no in situ observations were available. Genthon and Krinner [2001] have questioned the accuracy of the interpolation because it disagrees with the output of several numerical models of Antarctic precipitation and evaporation. It is uncertain whether the models share systematic errors or if the interpolation of the in situ observations is inaccurate. Huybrechts et al. [2000] produced a map using similar methods to Vaughan et al. [1999], including some additional in situ observations.

[8] We will compare several different approach to the interpolation of Antarctic in situ observations. These include (1) straightforward geostatistical interpolation of the accumulation rate observations, (2) guiding this interpolation using the correlation of accumulation rate with saturation vapor pressure [Robin, 1977], (3) guiding the interpolation using satellite microwave observations that are sensitive to layering within the snow-cover, and (4) a combined model that includes temperature and microwave observations. Our microwave method is a development of one used by Winebrenner et al. [2001] to map accumulation rates for the dry snow region of Greenland. This method makes use of satellite observations of thermal microwave emission, at $4.5-\mathrm{cm}$ wavelength, much longer than the $35 \mathrm{GHz}$ microwave observations used by Vaughan et al. [1999]. Because of the longer wavelength, scattering by grains is less important, and reflections that occur within the layered snow have the dominant effect upon the thermal emission [West et al., 1996]. In this paper, we investigate how the polarization induced by these reflections varies in Antarctica and relate those variations to the accumulation rate.

[9] Because of the variations of weather and climate that occur while it is deposited, the snow-cover of an ice sheet consists of many layers of high and low density snow interspersed [Colbeck, 1991]. The layering polarizes thermal microwave emission from the snow by acting as a reflective filter that preferentially transmits vertically polarized emission to the satellite and preferentially reflects horizontally polarized back to the depths [West et al., 1996]. Detecting the polarization using a satellite-borne radiometer allowed Winebrenner et al. [2001] to map the variation in this filtering effect across the dry snow region of the Greenland ice sheet. They used electromagnetic modelling and in situ observations collected from snowpits to relate this variation to the accumulation rate.

[10] Here, we extend the approach to investigate the combined effects of accumulation rate and temperature upon the polarization of thermal emission from Antarctica. We use observations of polarization recorded by the Advanced Microwave Scanning Radiometer (AMSR-E) on the Aqua satellite [Cavalieri and Comiso, 2004], and snow temperatures derived from satellite observations of thermal infrared emission under clear sky conditions [Comiso, 2000]. Figure 1 shows the location of in situ observations

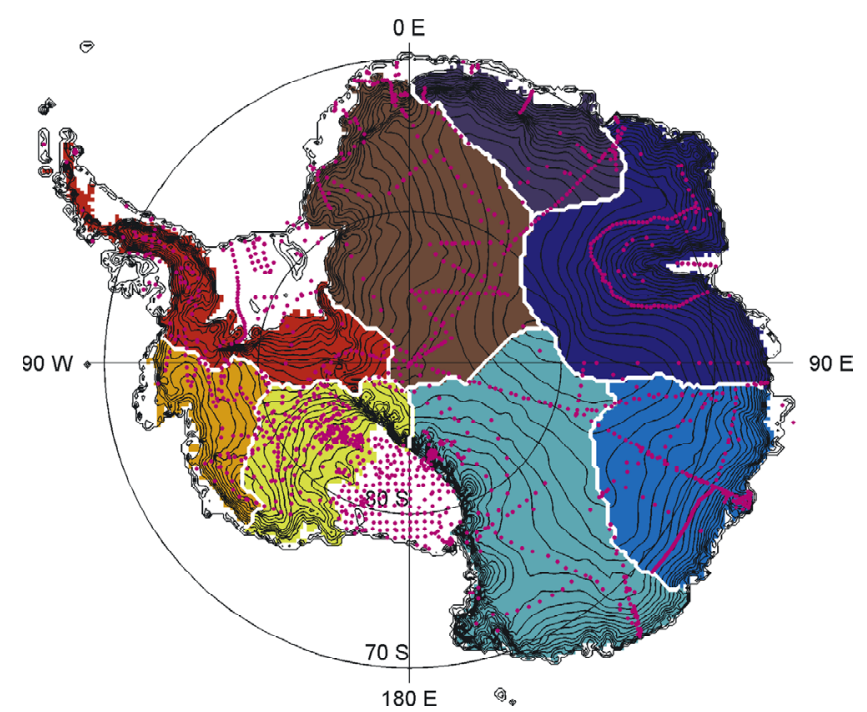

Figure 1. The location of in situ observations, and the major drainage sectors of Antarctica considered in this study.

of accumulation rate. We find a close relationship between the accumulation rate measured at these sites and the temperature and polarization derived from satellite observations. Our map of accumulation rate is derived by combining the satellite observations with the in situ observations using a geostatistical approach that accounts properly for spatial correlation among the observations.

[11] Our approach to interpolation allows a more objective estimate of errors than has been possible for previous studies. We pay particular attention to how these errors will affect spatial integrations used to compute the ice budget [Rignot and Thomas, 2002]. Figure 1 shows the areas draining into eight sectors of Antarctic coastline, assuming that ice flow is in the direction of steepest descent, which was computed using a digital elevation model of Antarctica [Bamber and Bindschadler, 1997]. We will estimate the spatially averaged accumulation rate for each of these drainage sectors and evaluate confidence intervals for these estimates.

\section{Method}

[12] We could simply interpolate the in situ observations using geostatistical interpolation method such as "ordinary kriging" [Kitanidis, 1997]. However, the observations are fairly sparse, and their interpolation can be assisted by choosing a suitable "background" field that approximates the spatial variations in the data [Vaughan et al., 1999]. Ordinary kriging uses a spatially uniform background field. Another possibility is to make use of the known correlation between accumulation rate and saturation vapor pressure [Robin, 1977], which varies strongly with temperature according to the Clausius-Clapeyron equation. Then we might choose as a background field

$$
a \approx \exp \left(c_{1}+c_{2} / T\right)
$$

where $a$ is accumulation rate, and $T$ is the mean annual surface temperature which is known reasonably well for 
Antarctica [Comiso, 2000]. We might expect differences between the mean annual surface temperature and the atmospheric temperatures that control snowfall. Selecting the parameters $c_{1}$ and $c_{2}$ using the in situ observations of accumulation rate, rather than thermodynamic theory, can compensate for this to some degree.

[13] In this paper we show that a much better choice of background field can be obtained by including microwave observations from the AMSR-E satellite radiometer that are sensitive to the layering within the snow-cover [West et al., 1996].

[14] AMSR-E observes thermally emitted microwave radiation at approximately $55^{\circ}$ from vertical. Two channels operate at $6.9 \mathrm{GHz}$, which corresponds to a wavelength of $4.3 \mathrm{~cm}$ in free space. The two channels measure the brightness temperatures $T_{B}(V)$ at vertical polarization and $T_{B}(H)$ at horizontal. The polarization ratio

$$
P=\frac{T_{B}(V)-T_{B}(H)}{T_{B}(V)+T_{B}(H)}
$$

quantifies the polarization effect, and therefore provides an indirect measure of layering in the snow [West et al., 1996].

[15] Winebrenner et al. [2001] analyzed data from the Scanning Multichannel Microwave Radiometer, a forerunner to AMSR-E. Comparing these data with in situ observations from the dry snow region of Greenland, they found that polarization $P$ was higher for sites with low accumulation rate $a$.

[16] Winebrenner et al. [2001] investigated the reasons for the relationship between polarization $P$ and accumulation rate $a$ by analyzing density profiles from snowpits. The snowpit profiles show that the density stratification at sites with high accumulation rate tends to have less variance on scales similar to the wavelength [Winebrenner et al., 2001]. Density stratification on this scale should have a strong control on polarization, according to numerical simulations of the reflection and transmission of thermally emitted microwave radiation within the snow deposit [West et al., 1996; Winebrenner et al., 2001]. In the simulations the snow-cover was modeled as a stack of layers, with density and thickness selected at random for each layer, to produce realistic density profiles. The simulations predict that greater variation in layer densities (on the wavelength scale) will increase polarization for the low accumulation rate sites above that of high accumulation sites, and this is what is observed. The simulations also show that thin, high-density crusts, a few millimeters thick, can influence the polarization considerably [Surdyk and Fily, 1995; Winebrenner et al., 2001].

[17] Aside from randomly varying deposition densities, another potential source of density layering is seasonal. Density differences between summer and winter snow layers are commonly observed in polar regions. In central Greenland, seasonal density variations are neither large enough nor sharp enough to contribute effectively to the polarization at $4.5 \mathrm{~cm}$ wavelength [Winebrenner et al., 2001]. On the Antarctic plateau, accumulation rates are much lower, so the annual layer thickness approaches the scale of the wavelength. Periodic density stratification on this scale would generate reflections and polarization. However, for such small annual layer thickness $(\leq 10 \mathrm{~cm})$, reworking of the snow by the wind after deposition probably eliminates much of the systematic density contrast between summer and winter layers, leaving a much more disordered layering.

[18] To summarize, a combination of random centimeterscale layering and thin millimeter-scale crusts probably controls the polarization, perhaps with some additional influence from seasonal layering. A predictive model of how accumulation rate affects the development of snow layering and metamorphism on scales of millimeters and centimeters is presently beyond us. Instead, we shall investigate a simple empirical model in which polarization ratio decreases with increasing accumulation rate as a power law $a^{-q}$, with exponent $q$ yet to be determined. In addition, we expect a component $P_{0}$ of the polarization to arise from reflection at the air-snow interface as thermal emission leaves the snow. One possible model for the polarization then takes the form

$$
P=P_{0}+k_{0} a^{-q}
$$

where the first term describes the polarization of surface reflection, and the second describes the influence of snow layering upon the polarization, parameterized as a function of mean annual accumulation rate $a(\mathbf{x})$. Defining $n=1 / q$, $\kappa \equiv k_{0}^{n}$, this suggests the following choice of background field for the accumulation rate

$$
a \approx \frac{\kappa}{\left(P-P_{0}\right)^{n}} .
$$

Many of the sintering and vapor transport processes that generate layering and crusts within the snow [Colbeck, 1991] proceed at rates that vary strongly with temperature according to Arrhenius-type relationships $\exp (-\vartheta / T)$. To allow for this effect, we shall also investigate a modified empirical model for the polarization which takes the form

$$
P=P_{0}+k_{0} a^{-q} e^{-\vartheta / T}
$$

with constant parameters $k_{0}, q$, and $\vartheta$. Again, the first term describes the polarization of surface reflection, but now the influence of snow layering is parameterized as a function of mean annual accumulation rate $a(\mathbf{x})$ and temperature $T(\mathbf{x})$. A strong temperature dependence may also arise if sporadic summer melting produces reflective crusts within the snow-cover. We expect that equation (5) would capture this variability better than equation (3). Note that the optimum choice of $k_{0}$, and possibly $q$, will be different for equation (5) than for equation (3).

[19] Rearranging equation (5) and defining $n=1 / q, \kappa \equiv$ $k_{0}^{n}$ and $\theta \equiv \vartheta n$ gives the following background field for the accumulation rate

$$
a \approx \frac{\kappa e^{-\theta / T}}{\left(P-P_{0}\right)^{n}} .
$$

In this paper we shall investigate four background fields of increasing complexity: ordinary kriging (constant background); the saturation vapor pressure background defined by equation (1); the polarization only background defined by equation (4); and the combined temperature and 


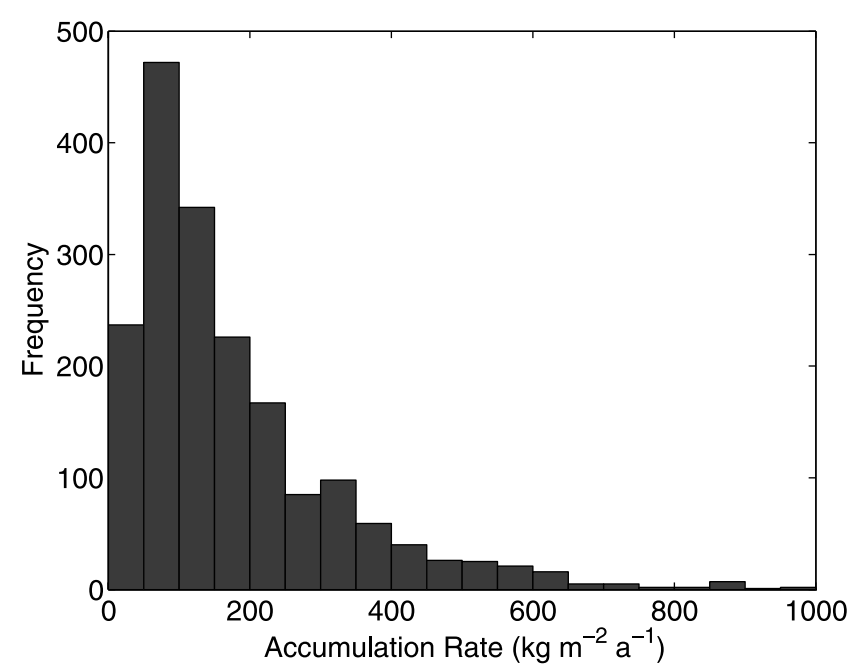

Figure 2a. The frequency distribution of in situ observations of accumulation rate $a_{i}\left(\mathrm{~kg} \mathrm{~m}^{-2} \mathrm{a}^{-1}\right)$.

polarization model defined by equation (6). All of these background fields can be viewed as special cases of equation (6), with either $\theta=0, n=0$, or both. Here we analyze the performance of the simpler models in parallel, to guard against any overelaboration that is not warranted by the in situ observations.

[20] We wish to be clear that we are undertaking an empirical study: the functional form of (5) is not predicted by any detailed theory, and other relationships between accumulation rate, temperature and polarization ratio, could of course be imagined. As it turns out, a suitable choice of parameters $\kappa, n$, and $\theta$ allows (6) to describe the relationship between temperature, accumulation rate and polarization ratio quite well. In fact, we show in section 4 that this relationship can explain nearly all the variance in the in situ observations, except for a component which occurs on short distance scales (less than $100 \mathrm{~km}$ ). On the basis of this observation we will ultimately use equation (6) as our background field to support the interpolation of the in situ observations.

[21] Mapping accumulation rate using equation (6) requires us to evaluate all quantities on the right-hand side. Polarization ratio $P(\mathbf{x})$ is available from AMSR-E observations [Cavalieri and Comiso, 2004], except at latitudes near the South Pole that are excluded from the satellite orbit. $T$ can be estimated using satellite observations of thermal infrared emission under clear sky conditions [Comiso, 2000].

[22] We estimated $P_{0}$ by computing the polarization of reflection at the air snow-interface for typical surface snow densities. We obtained a mean value of $P_{0}=0.035$ by computing horizontally and vertically polarized Fresnel reflection coefficients for over 1000 near-surface observations of density from Antarctic snowpits [Goodwin, 1988; Long, 1961; Pirrit and Doumani, 1961; Stuart and Heine, 1961]. We used expressions from Matzler [1996] to relate the density to electromagnetic permittivity. The sample standard deviation in $P_{0}$ computed from these density observations was 0.005 , negligible compared to spatial variations in total polarization $P$. We therefore fix $P_{0}=0.035$.

[23] The parameters $\kappa, \theta$ and $n$ still need to be determined. We leave them as free parameters to be estimated during the interpolation of the available in situ observations of accumulation rate $a_{i}(i=1,2 \ldots N)$. This approach allows us to use equation (6) as a background field to constrain the interpolation, while recognizing that we have no compelling reason a priori to adopt particular values for $\kappa, \theta$ and $n$.

\section{Universal Kriging}

[24] The interpolation and parameter estimation problem becomes more straightforward if we solve for the logarithmically transformed accumulation rate $y(\mathbf{x})$, defined as

$$
y \equiv \ln a
$$

The transformation has two advantages: (1) it greatly simplifies interpolation because the frequency distribution of $\ln a_{i}$ more closely resembles a Gaussian than the asymmetric distribution of untransformed accumulation rate $a_{i}$ (Figures $2 \mathrm{a}$ and $2 \mathrm{~b}$ ), so we can use standard least-squares methods; and (2) it allows us to optimize the interpolation and parameter estimation using the geostatistical method of universal kriging [Kitanidis, 1997].

[25] Essentially, we can write

$$
y=b+\varepsilon, \quad \text { with } b \equiv \sum_{j=1}^{J} c_{j} X_{j} .
$$

The field $\varepsilon(\mathbf{x})$ is the residual from a background field $b(\mathbf{x})$ represented as a linear combination of known spatial patterns $X_{j}(\mathbf{x})$ each of which is multiplied by an amplitude coefficient $c_{j}$. Universal kriging allows the estimation of $y(\mathbf{x})$, given knowledge of the patterns $X_{j}$ and a vector of observations $\mathbf{z}$. The method simultaneously solves for the coefficients $c_{j}$ that give the closest agreement between the background field and the observations.

[26] In our case, the elements of the observation vector $\mathbf{z}$ are the transformed in situ accumulation rate measurements $z_{i}=\ln a_{i}$, so we have

$$
z_{i}=y\left(x_{i}\right)+\delta_{i}
$$

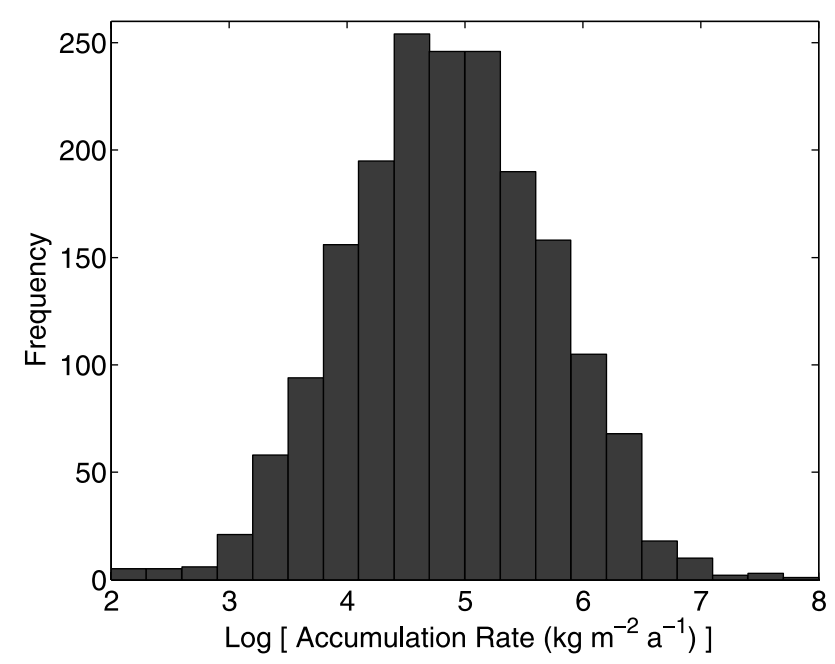

Figure 2b. The frequency distribution of the logarithm $\ln a_{i}$ of in situ observations of accumulation rate $a_{i}\left(\mathrm{~kg} \mathrm{~m}^{-2} \mathrm{a}^{-1}\right)$. 
with $\delta_{i}$ representing the effects of measurement error, temporal variability, and short-scale spatial variability.

\section{Choosing a Background Model}

[27] We investigated four choices of background field. The first,

$$
b=c_{1},
$$

is constant everywhere and corresponds to ordinary kriging of the logarithmically transformed observations. The second choice of background,

$$
\begin{gathered}
b=c_{1}+c_{2} X_{2}, \\
X_{2} \equiv \frac{1}{T},
\end{gathered}
$$

allows for variations of accumulation rate with temperature but makes no use of the polarization observed by the AMSR-E radiometer. Allowing for the logarithmic transformation we have $a \approx \exp \left(c_{1}+c_{2} / T\right)$, so this choice of background corresponds to using the saturation vapor pressure model defined by equation (1).

[28] The third choice of background uses polarization only

$$
\begin{gathered}
b=c_{1}+c_{3} X_{3}, \\
X_{3} \equiv \ln \left(P-P_{0}\right) .
\end{gathered}
$$

This corresponds to using (4) as a background model.

[29] Fourth, and most generally, we use the logarithm of equation (6) as a background field, which gives

$$
b=c_{1}+c_{2} X_{2}+c_{3} X_{3} .
$$

In each case, the $X_{j}$ are determined entirely by satellite observations and other known quantities $\left(P(\mathbf{x}), T(\mathbf{x}), P_{0}\right)$. The coefficients are left as free parameters to be determined from the in situ observations, so the values of $c_{1}, c_{2}$ and $c_{3}$ vary depending upon which background model is used. For the most general model (13), we have

$$
c_{1} \equiv \ln \kappa, \quad c_{2} \equiv-\theta, \quad c_{3} \equiv-n,
$$

so applying universal kriging, with (13) as the background field, automatically selects the best values for the unconstrained parameters $\kappa, \theta$, and $n$ (i.e., those values that minimize discrepancies with the in situ observations).

[30] We characterize the spatial statistics of departures from each of the background fields by computing their semivariogram [Kitanidis, 1997]. For now, we assume that these statistics are homogenous and isotropic. The semivariogram of residuals is then defined as

$$
\gamma_{z z}\left(h_{i j}\right) \equiv \frac{1}{2}\left\langle\left\{\left[z_{i}-b\left(\mathbf{x}_{i}\right)\right]-\left[z_{j}-b\left(\mathbf{x}_{j}\right)\right]\right\}^{2}\right\rangle,
$$

where angled brackets represent expected quantities, and the locations $\mathbf{x}_{i}$ and $\mathbf{x}_{j}$ are separated by a distance $h_{i j}=\left|\mathbf{x}_{i}-\mathbf{x}_{j}\right|$.

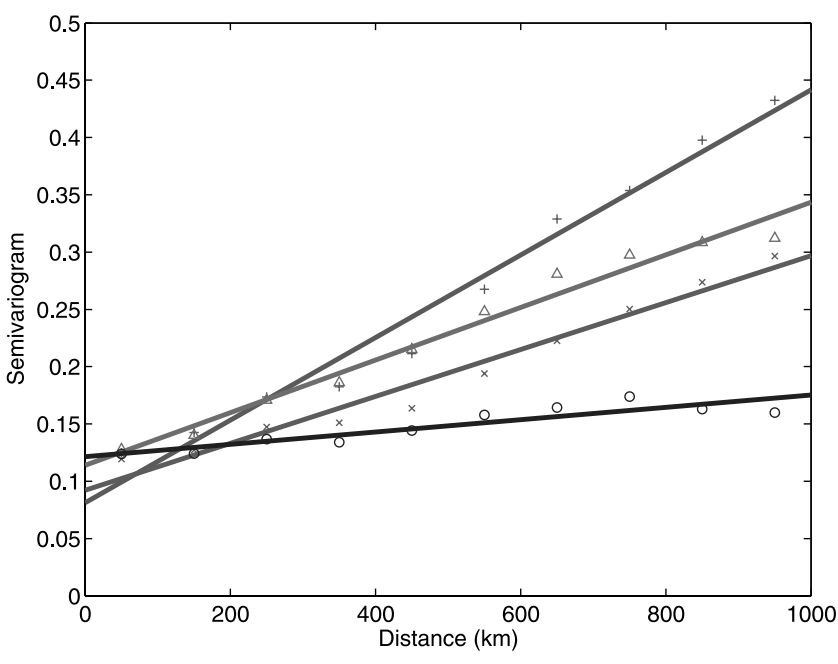

Figure 3. The semivariogram of the logarithmically transformed in situ observations of accumulation rate $\ln a_{i}$ $(+)$, and of the residuals of $\ln a_{i}$ from the background models defined by equations (11), shown by triangles; (12), shown by crosses; and (13), shown by circles.

[31] For each background model, Figure 3 shows an empirical approximation to $\gamma_{z z}(h)$. These were evaluated at a set of evenly spaced distance intervals using the method described in Appendix A. All four semivariograms are well approximated by linear models of the form

$$
\gamma_{z z}(h)=\alpha+\beta h, \quad h>0 .
$$

The intercept $\alpha$ is similar in all cases and represents the combined contribution to the variance of $\ln a_{i}$ made by measurement errors, any temporal variations that decorrelate between measurements, and short-scale spatial variability. Clearly, none of the background models can capture this component of the variation.

[32] The intercept $\alpha=0.1$ corresponds to r.m.s. shortscale variability of about $\pm 30 \%$ in $a_{i}$, when the logarithmic transformation is taken into account. This is comparable to the short-scale spatial variability $(20 \%-60 \%)$ estimated independently using ground-penetrating radar [Richardson et al., 1997].

[33] Turning to the broader-scale patterns, the linear term $\beta h$ in the semivariogram shows that residuals $\varepsilon(\mathbf{x})$ exhibit ever larger fluctuations at increasing spatial scales. The semivariograms do not reach a plateau or sill at any scale, so there seems to be no preferred length scale for relative variations in the accumulation rate with respect to any of the background models.

[34] The semivariogram for the constant-background model (10) has the largest gradient $\beta$. By contrast, when we use the temperature-compensated polarization model (13), derived from equation (6), the gradient $\beta$ approaches zero. This shows that the background field defined by (13) captures nearly all of the variation on scales larger than $100 \mathrm{~km}$. When either temperature or polarization are ignored, as in equations (1) and (3), the background field captures only about half of the broad-scale variability. We conclude that a combination of temperature and polarization 
Table 1. Temporal Coverage of in Situ Data By Decade

\begin{tabular}{lc}
\hline \multicolumn{1}{c}{ Decade } & Percentage \\
\hline Pre-1950 & $1 \%$ \\
$1950-1960$ & $13 \%$ \\
$1960-1970$ & $17 \%$ \\
$1970-1980$ & $19 \%$ \\
$1980-1990$ & $49 \%$ \\
$1990-2000$ & $1 \%$ \\
\hline
\end{tabular}

is necessary to capture the majority of the variability in accumulation rate on scales greater than $100 \mathrm{~km}$.

\section{Producing a Map}

[35] It is important to understand that we are not seeking to map variations in accumulation rate at very fine spatial scales. The brightness temperatures recorded by the AMSR radiometer are representative of a footprint on the ground that is roughly $60 \mathrm{~km}$ in diameter. Given the sparsity of the in situ measurements, the highest spatial resolution we can hope for is comparable with the dimension of the satellite footprint. Since our main interest is in deriving spatial averages of accumulation rate across drainage basins, the averaging performed by the satellite is advantageous. The semivariograms show that point measurements are likely to suffer from $30 \%$ r.m.s. short-scale variations when compared to the broad-scale (e.g., $10^{4} \mathrm{~km}^{2}$ ) average accumulation rate in the region where the measurements are taken. Clearly, it is desirable to optimize the interpolation so that the short-scale variability does not unduly influence our map.

[36] Similar considerations apply to temporal averaging. The satellite observations of microwave polarization are sensitive to layering in the snow that has been deposited over many hundreds, even thousands, of years, so they represent a long-term average. The temperature observations were averaged over the interval 1982-1997 but can be expected to approximate the long-term mean temperatures fairly closely, since these are controlled to a great degree by the elevation of the surface. The most important consideration is that we have chosen the parameters that best fit the in situ observations.

[37] Table 1 shows the frequency distribution of the 540 in situ observations for which start and end dates are reported by Vaughan and Russell [1997]. In computing Table 1, measurements spanning more than 1 decade were distributed in proportion to the fraction of the total measurement interval that lay within each decade. Broadly speaking, the in situ observations sample accumulation that fell as snow between 1950 and 1990.

[38] To produce a map of the annual-mean snow accumulation, we must reduce the impact of measurement errors, and temporal variability as best we can. It is impossible to resolve the short-scale spatial variability using the measurements available to us. Rather than force our map to reproduce every observation we therefore aim only to represent the broad-scale accumulation patterns. We do this by the method of continuous-part universal kriging as described by Kitanidis [1997]. In this approach, a filter is designed that optimally smooths out the variability that contributes to the intercept $\alpha$ of the semivariogram, leaving the best approximation to the longer-wavelength spatial variations. We defer the details of this to Appendix B.

[39] The outcome of the estimation procedure is a set of accumulation rate estimates $\hat{a}$ for each cell of a rectangular grid in polar stereographic coordinates (defined by the WGS84 ellipsoid, and latitude of true scale at 71S).

[40] We also consider averages over the major drainage sectors of Antarctica (Figure 1). We represent the areal integration of accumulation over some region through a simple numerical quadrature defined by

$$
\hat{A}=\mathbf{H} \hat{a},
$$

with elements of the row vector $\mathbf{H}$ being equal to the area represented by the grid cell if that cell is to be included in the integration, and zero otherwise. More sophisticated integration schemes could also be written in this form.

[41] Continuous-part universal kriging is an example of best linear unbiased estimation (BLUE), for which wellknown error estimates are available under the assumption that measurement errors, and departures from the background field, have Gaussian statistics. The details of the error analysis are presented in Appendix C.

[42] One might argue that the preliminary background field would itself be a useful map of accumulation rate, without resorting to the kriging approach. We have retained the kriging step, however, for three reasons. (1) The slope of the semivariogram (though much reduced for our chosen background field) is not eliminated entirely, so there remain some residual broad-scale patterns that can be removed by interpolation. (2) By using the background as a guide, rather than a strong constraint, our map will locally defer to nearby observations (which may be confirmed by direct inspection of how individual measurements are being weighted). This capacity for local adjustment might be important in regions where the microwave observations are affected by atypical processes such as melting and refreezing. (3) By accounting properly for spatial corellation, we expect to obtain better estimates of model parameters than from a straightforward unweighted least squares fit.

\section{Results}

[43] Figure 4 shows the map of accumulation rate. The main features are similar to previous compilations [Vaughan et al., 1999; Giovinetto and Zwally, 2000; Huybrechts et al., 2000]. Generally speaking, high accumulation in coastal regions gives way to very low accumulation rates in the interior of the continent. In coastal regions of East Antarctica, slopes that face east typically receive higher accumulation than slopes that face west.

[44] On closer inspection there are differences between the new map and earlier ones. Figures 5 and 6 show differences between the new map and the earlier compilation of Vaughan et al. [1999]. There are regions where differences exceed $100 \mathrm{~kg} \mathrm{~m}^{-2} \mathrm{a}^{-1}$. Since the same in situ observations were used for both maps, these differences must arise from the different approaches to interpolation. Our analysis suggests that the compilation of Vaughan et al. [1999] overestimates accumulation rates in the sector $90-120^{\circ} \mathrm{E}$, and underestimates accumulation in Marie Byrd Land $210-250^{\circ} \mathrm{W}$, and east of the Amery Ice Shelf 


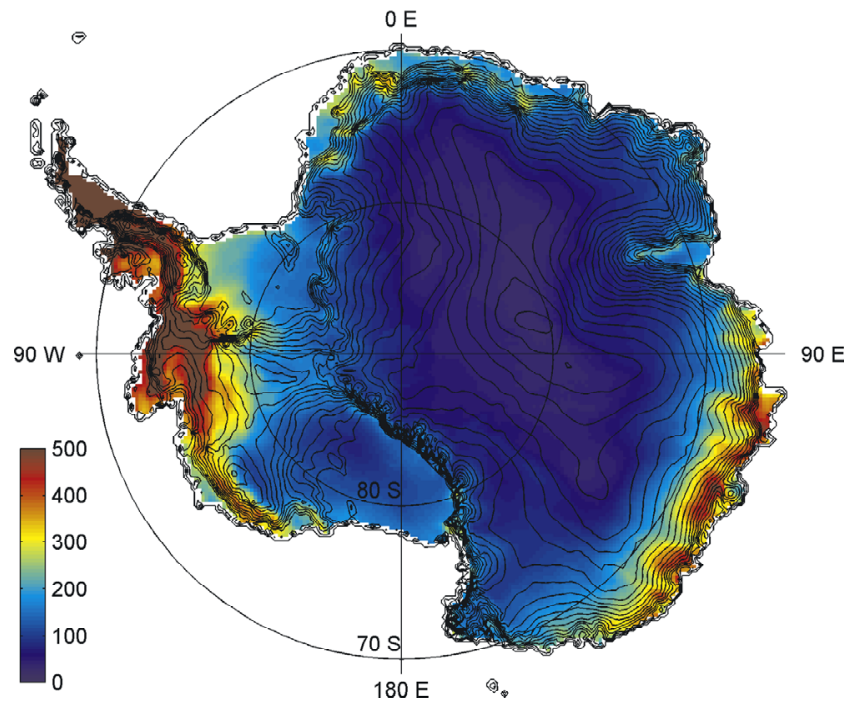

Figure 4. The map of Antarctic snow accumulation rate derived in this study $\left(\mathrm{kg} \mathrm{m}^{-2} \mathrm{a}^{-1}\right)$.

70-90 ${ }^{\circ}$ E. Genthon and Krinner [2001] obtain a similar pattern of differences between a composite of numerical atmospheric models and the Vaughan et al. [1999] map, which suggests that the new map may eliminate some of the discrepancy between model results and compilations of in situ data. Figure 5 shows differences between the new map and the earlier compilation of Giovinetto and Zwally [2000]. Again the general pattern of accumulation is very similar for the two maps, but there are regions where differences exceed $100 \mathrm{~kg} \mathrm{~m}^{-2} \mathrm{a}^{-1}$. Our map has lower accumulation over Thwaites drainage basin than either Vaughan et al. [1999] or Giovinetto and Zwally [2000] which suggests that Rignot and Thomas [2002] may have underestimated the net loss of ice to the ocean from this region. Detailed comparisons of the new map with measurements of the ice-flux drained by the major outlet glaciers will be needed to assess the state of balance

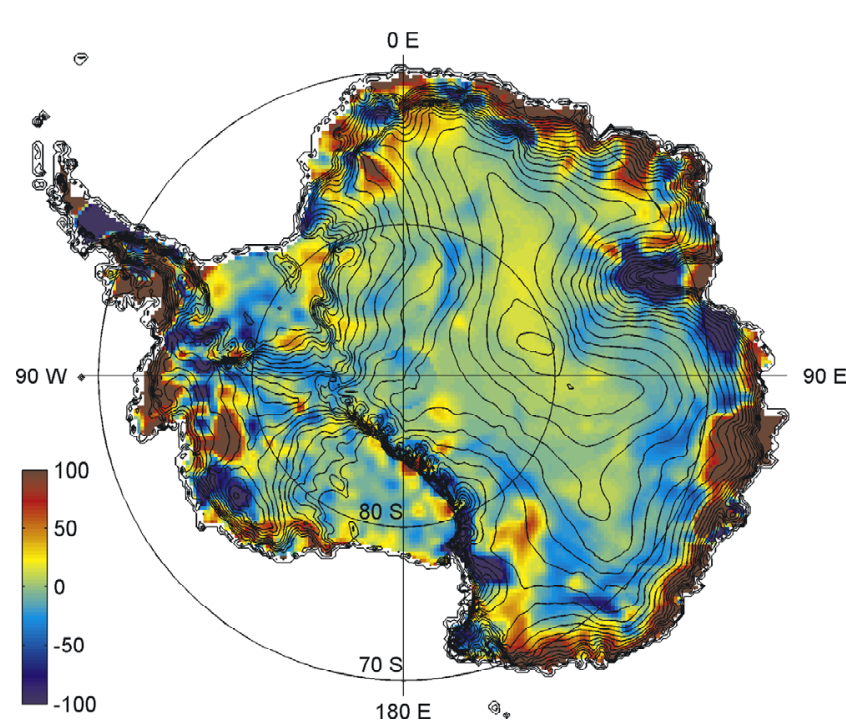

Figure 5. Differences obtained by subtracting Figure 4 from Vaughan et al. [1999] $\left(\mathrm{kg} \mathrm{m}^{-2} \mathrm{a}^{-1}\right)$.

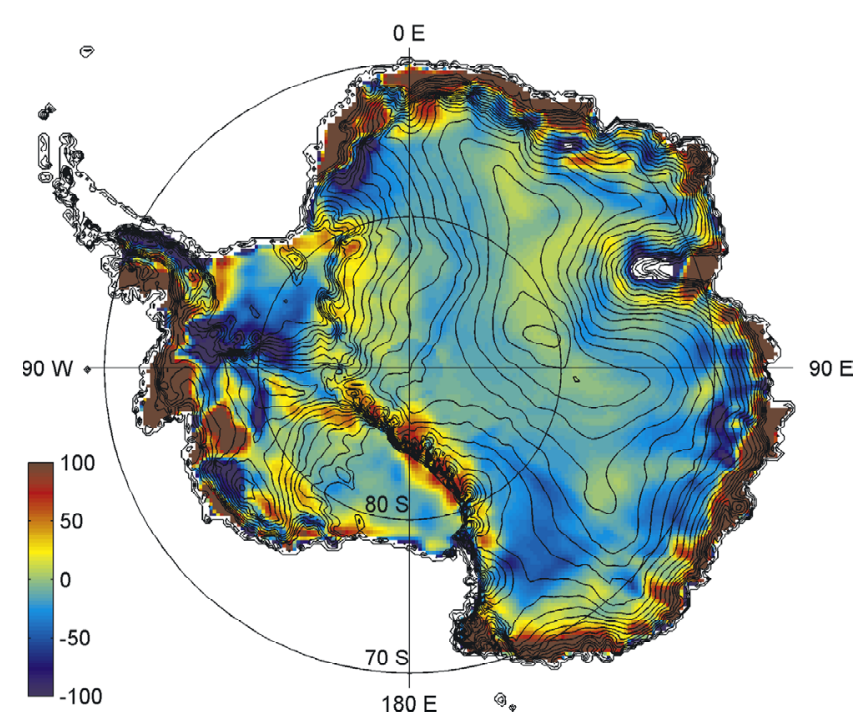

Figure 6. Differences obtained by subtracting Figure 4 from Giovinetto and Zwally [2000] $\left(\mathrm{kg} \mathrm{m}^{-2} \mathrm{a}^{-1}\right)$.

of various regions, and how this differs from estimates derived using the earlier maps of accumulation rate.

[45] The error analysis described in Appendix C allows us to estimate the root mean square error in the new map. This is plotted in Figure 7, as a percentage of the mapped value at each location. Generally, the r.m.s. errors are lower than $10 \%$. This means that we would expect a full survey of the average accumulation within a region about $10^{4} \mathrm{~km}^{2}$ in area to agree with our map to this precision. We know from our earlier analysis that any individual snowpit or ice core will disagree by about $30 \%$ r.m.s. because of the short-scale spatial variations.

[46] The value of including the satellite observations is demonstrated by performing an identical analysis using the constant background field (10) in place of equation (13). Without the satellite information, there would be regions

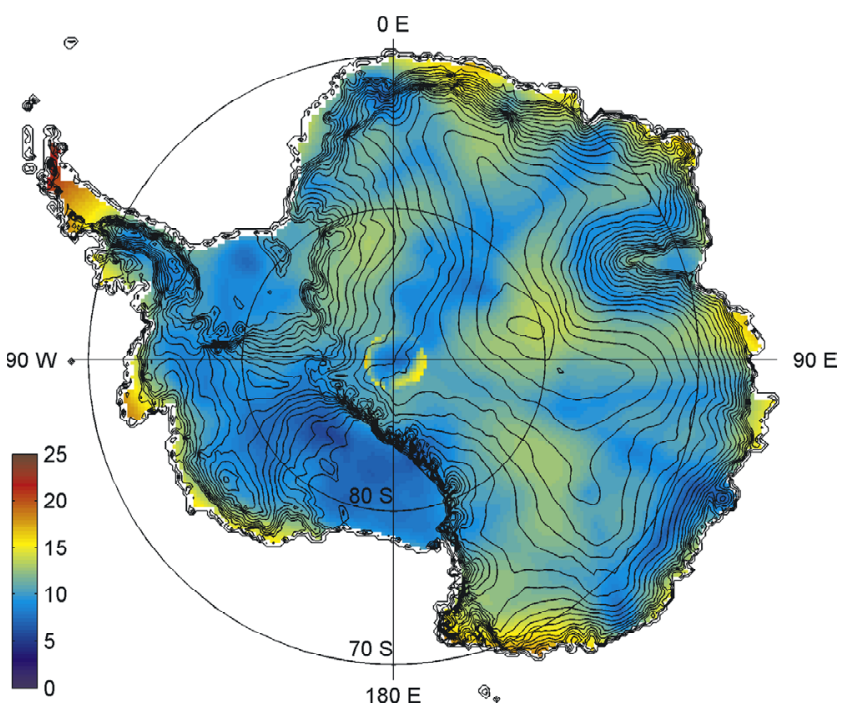

Figure 7. Estimated root mean square error for the accumulation map shown in Figure 4, expressed as a percentage. 
Table 2. Area, Accumulation Rate, and Percentage Root Mean Square Error for the Drainage Sectors Shown in Figure 1

\begin{tabular}{lccc}
\hline \multicolumn{1}{c}{$\begin{array}{c}\text { Sector } \\
0-45 \mathrm{E}\end{array}$} & Area, $\mathrm{m}^{2}$ & $\begin{array}{c}\text { Accumulation } \\
\text { Rate, } \mathrm{kg} \mathrm{m}^{-2} \mathrm{a}^{-1}\end{array}$ & $\begin{array}{l}\text { RMS } \\
\text { Error }\end{array}$ \\
$45-95 \mathrm{E}$ & $8.72 \times 10^{11}$ & 112 & $5.6 \%$ \\
$95-135 \mathrm{E}$ & $21.1 \times 10^{11}$ & 107 & $3.5 \%$ \\
$135-180 \mathrm{E}$ & $13.8 \times 10^{11}$ & 194 & $2.8 \%$ \\
$180-225 \mathrm{E}$ & $26.8 \times 10^{11}$ & 106 & $3.4 \%$ \\
$225-275 \mathrm{E}$ & $9.43 \times 10^{11}$ & 147 & $2.8 \%$ \\
$275-315 \mathrm{E}$ & $5.82 \times 10^{11}$ & 364 & $4.2 \%$ \\
$315-360 \mathrm{E}$ & $9.97 \times 10^{11}$ & 308 & $3.7 \%$ \\
$0-360 \mathrm{E}$ & $25.4 \times 10^{11}$ & 77 & $2.8 \%$ \\
\hline
\end{tabular}

of large error (up to $40 \%$ r.m.s.) far from the in situ observations.

[47] Spatial averages of the accumulation rate for the drainage sectors shown in Figure 1 are provided in Table 2. The errors for spatial averages are derived in Appendix C. These error estimates are also listed in Table 2. Assuming normally distributed errors, and doubling r.m.s. errors to give approximate $95 \%$ confidence intervals, we estimate the precision of these spatial integrations at $5-10 \%$.

[48] Table 2 also shows the value obtained by averaging the new map over all regions of grounded ice, $143 \pm 4 \mathrm{~kg} \mathrm{~m}^{-2} \mathrm{a}^{-1}$. This is slightly lower than the estimate of $149 \mathrm{~kg} \mathrm{~m}^{-2} \mathrm{a}^{-1}$ obtained by Church et al. [2001] as an average of studies published since 1995, but lies within the range of variability of those studies. We also repeated our analysis, just using the 546 in situ measurements for which Vaughan and Russell [1997] report start and end dates for the measurement intervals (see Table 1). This changed the average accumulation by less than $2 \%$, so we consider the average snowfall derived from the map to be broadly representative of the epoch 19502000. It is true though that field surveys have concentrated upon different basins at different times. A fuller examination of temporal fluctuations in accumulation rate, and the spatiotemporal sampling of those fluctuations by the various field campaigns that have operated in Antarctica would be useful, especially if temporal changes in accumulation rate are to be investigated by comparing our map with future versions.

\section{Discussion}

[49] The functional form of equation (5) was not predicted by any detailed theory of snow deposition or metamorphism. Nevertheless, the parameters recovered by universal kriging may provide some insight into the dependence of polarization (and by implication the processes that control snow stratification) upon temperature and accumulation rate. We estimate the value of $q$ as $1.9 \pm 0.3$, so polarization ratio caused by layering varies approximately as the inverse square of accumulation rate. We estimate the parameter $\vartheta$ as $3500 \pm 800 \mathrm{~K}$. This corresponds to a temperature dependence of the form $\exp (-E / R T)$ with $R \equiv 8.314 \mathrm{~J} \mathrm{~K}^{-1} \mathrm{~mol}^{-1}$ being the gas constant, and activation energy $E \approx 29 \pm 7 \mathrm{~kJ} \mathrm{~mol}^{-1}$, of comparable magnitude, but somewhat lower, than the temperature dependence of sintering estimated from empirical observations of snow compaction in the upper layers of Antarctic firn [Alley, 1987; Kojima, 1964].

[50] It is impossible to rule out fortuitous correlations between temperature, accumulation rate, and other factors that promote stratification, such as wind, solar radiation, or sporadic melting. Thus we do not suggest that wind, melt, or radiation are not important in determining the stratification of Antarctic snow, only that, if they are important, their effect upon the polarization can successfully be described in terms of mean annual temperature and accumulation rate for a wide variety of snow types and climatic conditions. Models of the depositional and metamorphic processes which generate density stratification and crusts have been developed and tested for Alpine conditions [e.g., Lehning et al., 2002]. Similar models could be used to investigate the importance of individual processes upon the stratification under Antarctic conditions.

[51] Although we do not have a complete physical explanation for equation (6), it is quite successful at explaining the variation in accumulation rate across the Antarctic ice sheet. The compilation of in situ measurements is fairly extensive, so the background field defined by equation (6) need only provide limited assistance to interpolation. Our error analysis demonstrates that it is adequate for that purpose, providing useful information about accumulation rate in regions of the ice sheet that are poorly sampled by field surveys.

[52] We must add a caveat that few of the in situ observations used to derive the surface reflection $P_{0}$, or to constrain the background model, come from regions that are subject to significant melting and runoff, or to substantial meltwater percolation and refreezing within the snow. Melting has a strong effect upon the microwave scattering properties of the snow-cover, so values of net snow accumulation and the errors computed here are at best provisional for ablation and percolation zones and may well turn out to be inappropriate. Regions where such strong melting occurs make up a small areal fraction of Antarctica, mostly confined to peripheral ice shelves.

[53] The error estimates derived in Appendix $\mathrm{C}$ are based upon several assumptions. We have assumed that after logarithmic transformation, departures from the background field are statistically similar at all places and in all directions, so that the semivariogram is purely a function of separation. We have also assumed that the departures from the background field have a Gaussian distribution. These assumptions are common in geostatistical interpolation. Nevertheless, they have been forced upon us more by the limited number of in situ observations than by any stronger compulsion. Further in situ observations might reveal that departures from the background cannot be regarded as a homogenous, isotropic, Gaussian field. In that case our error estimates would need to be revised, and more elaborate inversion methods would be needed to produce a map. The assumptions we have made here are perhaps most suspect near sharp linear boundaries between high and low accumulation. Meteorological effects can cause sudden jumps in accumulation rate across ice divides so that departures from the smooth background field might locally be bimodal, rather than Gaussian, and the departures from the smooth background field might also become anisotropic and inhomogenous. In any case, the limited resolution of the satellite, and the sparsity of the field observations, means that jumps in accumulation rate across divides are likely to be much smoother in our map than in reality. The effect of this smoothing upon calculations of ice budget 
for individual drainage basins should be considered, since it may apparently transfer snowfall from higher accumulation basins to lower accumulation basins. Given that we expect this smoothing to operate over a scale of $100 \mathrm{~km}$ or so, we would recommend that basins are combined to achieve dimensions of order $1000 \mathrm{~km}$, and areas of order $10^{6} \mathrm{~km}^{2}$, for computations of the ice budget.

\section{Conclusions}

[54] We have carried out an empirical investigation into the relationship between mean annual temperature, accumulation rate, and the polarization of thermally emitted microwaves. The microwave emission at $4.3 \mathrm{~cm}$ wavelength was viewed from a direction $55^{\circ}$ from the local vertical by the AMSR-E radiometer. We find that the polarization varies with accumulation rate according to an inverse square power law, and with temperature according to an Arrhenius type relationship with apparent activation energy $29 \pm 7 \mathrm{~kJ}$ $\mathrm{mol}^{-1}$. When we invert this relationship for the accumulation rate, it is able to explain most of the variation in in situ observations of accumulation rate on scales greater than about $100 \mathrm{~km}$. It therefore provides a suitable background field to support the interpolation of those observations.

[55] After a logarithmic transformation we performed the interpolation using continuous-part universal kriging, a linear estimation method that allows several of the parameters that define the background field to be estimated during interpolation. Continuous-part universal kriging removes the short scale spatial variability that contributes to the variance among measurements. The method is an example of best linear unbiased estimation (BLUE), a class of inverse methods for which estimates of the error may be derived, under the assumption that measurement errors and residuals from the background field have Gaussian distributions.

[56] We estimate the effective resolution of our map to be in the region of $100 \mathrm{~km}$. On spatial scales smaller than $10^{4} \mathrm{~km}^{2}$ we expect accumulation rates to vary significantly from the gridded values that we obtain from our universal kriging analysis.

[57] Averaging our map over the major drainage sectors of Antarctica, we are able to estimate the average accumulation rate to a precision of $10 \%$ or better. Averaging over the grounded ice of Antarctica, we estimate that from 1950 to 2000 the mean annual accumulation of snow in Antarctica was $143 \pm 4 \mathrm{~kg} \mathrm{~m}^{-2} \mathrm{a}^{-1}$.

\section{Appendix A: Estimation of the Semivariogram}

[58] The first step in computing the semivariogram is to use the observation vector $\mathbf{z}$ to solve for a rough approximation $\hat{\mathbf{b}}$ to the background field at the observations sites. For each in situ observation site $(i=1,2, \ldots, N)$ we define the $i$ th row of an $N \times 3$ matrix $\mathbf{X}_{z}$ as $\left[1,1 / T_{i}, \ln \left(P_{i}-P_{0}\right)\right]$, in which $P_{i}$ and $T_{i}$ are the polarization and temperature, determined from satellite observations. The rough approximation $\hat{\mathbf{b}}$ is then defined by $\hat{\mathbf{b}} \equiv \mathbf{X}_{z} \hat{\mathbf{c}}$, where $\hat{\mathbf{c}}$ is the unweighted least squares solution of $\mathbf{z}=\mathbf{X}_{z} \hat{\mathbf{c}}$. The empirical approximation to the semivariogram for a given distance interval is obtained by computing the sample mean of $\frac{1}{2}\left[\left(z_{i}-\hat{b}_{i}\right)-\left(z_{j}-\hat{b}_{j}\right)\right]^{2}$ for all pairs of observations $z_{i}$ and $z_{j}$ that are separated by distances within that interval. See Kitanidis [1997] for further details.

\section{Appendix B: Solving for Accumulation Rate}

[59] We define a rectangular grid of locations, then seek an estimate $\hat{a}$ of the average accumulation rate within each grid cell. The first step is to solve for $\hat{y}$, an estimate of the logarithmically transformed accumulation rate for each grid point, using the logarithmically transformed in situ measurements of accumulation rate $z_{i} \equiv \ln a_{i}$. Universal kriging is an application of best linear unbiased estimation (BLUE). We write the estimates as a linear combination of the observations,

$$
\hat{\mathbf{y}}=\Lambda \mathbf{z}
$$

with $\hat{\mathbf{y}}$ being a column vector composed of estimates $\hat{y}$ for all grid locations and $\Lambda$ being a matrix of weights to be determined. The weights $\boldsymbol{\Lambda}$ are chosen to satisfy the BLUE equation:

$$
\left[\begin{array}{cc}
-\gamma_{z z} & \mathbf{X}_{z} \\
\mathbf{X}_{z}^{T} & \mathbf{0}
\end{array}\right]\left[\begin{array}{l}
\boldsymbol{\Lambda}^{T} \\
\mathbf{M}
\end{array}\right]=\left[\begin{array}{c}
-\boldsymbol{\gamma}_{z y} \\
\mathbf{X}_{y}^{T}
\end{array}\right]
$$

In equation (B2), $T$ denotes the matrix transpose. The matrix $\gamma_{z z}$ depends upon the intercept and slope of the semivariogram. It has elements $\alpha\left(1-\delta_{i j}\right)+\beta h_{i j}$, with $h_{i j}$ being the distance between sites of the $i$ th and $j$ th in situ measurements and $\delta_{i j}$ being the Kronecker delta. The matrix $\gamma_{z y}$ has elements $\alpha+\beta h_{i k}$, where $h_{i k}$ is the distance between the $i$ th measurement and the $k$ th grid location. The matrix $\mathbf{X}_{z}$ is defined in Appendix A. The matrix $\mathbf{X}_{y}$ is defined similarly, but for grid locations, so its $k$ th row is $\left[1,1 / T_{k}, \ln \left(P_{k}-P_{0}\right)\right]$, with $P_{k}$ and $T_{k}$ evaluated from satellite observations at the $k$ th grid point. The system of equations (B2) is designed so that solving for the matrix of Lagrange multipliers $\mathbf{M}$ along with the weights $\Lambda$ ensures the solution defined by equation (B1) is unbiased, and gives a maximum likelihood solution for $\hat{\mathbf{y}}$ when errors and departures from the background field have a Gaussian distribution. For further details of best linear unbiased estimation and continuous-part universal kriging, see Kitanidis [1997].

[60] Once $\hat{y}$ has been determined using equation (B1) and equation (B2), the accumulation rate $\hat{a}$ is found by reversing the logarithmic transformation so that $\hat{a}=\chi \exp [\hat{y}]$. The multiplicative correction factor $\chi$ is needed because the three-step procedure (transformation, interpolation, reversetransformation) would otherwise lead to biased results. We determine $\chi$ by requiring that $\langle\hat{a}\rangle$ should equal $\left\langle\hat{a}_{i}\right\rangle$ at measurement locations, angled brackets denoting expected quantities.

[61] Suppose $a$ is the true accumulation averaged over the grid cell. Writing $z_{i}=\ln a+\delta$, and $\hat{y}=\ln a+v$ implies

$$
\frac{\left\langle a_{i}\right\rangle}{\langle\hat{a}\rangle}=\frac{\left\langle\exp z_{i}\right\rangle}{\langle\chi \exp \hat{y}\rangle} \approx \frac{\left\langle a\left(1+\delta+\frac{1}{2} \delta^{2}\right)\right\rangle}{\chi\left\langle a\left(1+\nu+\frac{1}{2} \nu^{2}\right)\right\rangle} .
$$


From which

$$
\chi=\frac{1+\frac{1}{2}\left\langle\delta^{2}\right\rangle}{1+\frac{1}{2}\left\langle v^{2}\right\rangle},
$$

corrects most of the bias introduced by nonlinear terms, when $\langle\delta\rangle=0,\langle\nu\rangle=0$, and $\delta$ and $\nu$ are small enough and approximately normally distributed. We use $\alpha$, the intercept of the semivariogram, as our estimate for $\left\langle\delta^{2}\right\rangle$. Our estimate of $\left\langle v^{2}\right\rangle$ is based on the error analysis of universal kriging (Appendix $C$ ). Since $\alpha \approx 0.1$, and is considerably larger than our estimates of $\left\langle v^{2}\right\rangle$, we obtain $\chi \approx 1.05$ everywhere, so applying this correction increases the accumulation rate by approximately $5 \%$.

\section{Appendix C: Errors}

[62] If $\mathbf{y}$ is the vector of log-transformed accumulation rate for all grid cells, the error in our estimate is $\nu \equiv \hat{\mathbf{y}}-\mathbf{y}$. Following Kitanidis [1997], the error covariance $\mathbf{C}_{\nu \nu} \equiv$ $\left\langle\nu \nu^{T}\right\rangle$, with angled brackets denoting expected quantities, is $\mathbf{C}_{\nu \nu}=-\mathbf{X}_{y} \mathbf{M}-\gamma_{y y}+\gamma_{z y}^{T} \Lambda^{T}$, where $\gamma_{y y}$ has elements $\alpha+$ $\beta h_{k l}$, and the distance $h_{k l}$ separates the $k$ th and $l$ th grid points. When the errors are small enough to neglect terms of order $O\left(v^{2}\right)$, Taylor series expansion of the logarithm (7) implies that $\mathbf{a} \approx \hat{\mathbf{a}}-\hat{\mathbf{a}}^{D} \boldsymbol{\nu}$, where $\hat{\mathbf{a}}^{D}$ is the square diagonal matrix with leading diagonal $\hat{\mathbf{a}}$. The absolute error in accumulation rate $\epsilon \equiv \hat{\mathbf{a}}-\mathbf{a}$ then has covariance $\mathbf{C}_{\epsilon \epsilon} \equiv$ $\left\langle\epsilon \epsilon^{T}\right\rangle$ approximately given by $\mathbf{C}_{\epsilon \epsilon} \approx \hat{\mathbf{a}}^{D} \mathbf{C}_{\boldsymbol{\nu} \boldsymbol{\nu}} \hat{\mathbf{a}}^{D}$. When equation (17) is used to form areal averages, and $\mathbf{A}$ represents the true accumulation integrated over the selected region, the variance of the error $\Delta \equiv \hat{A}-A$ is given by $\left\langle\Delta^{2}\right\rangle=\mathbf{H} \hat{\mathbf{a}}^{D} \mathbf{C}_{\boldsymbol{\nu} \boldsymbol{\nu}} \hat{\mathbf{a}}^{D} \mathbf{H}^{T}$.

[63] Acknowledgments. We thank three anonymous reviewers and the editors for their helpful suggestions and comments. DPW was partially supported by NSF grant 0126161 .

\section{References}

Alley, R. B. (1987), Firn densification by grain-boundary sliding: A first model, J. Phys., 48(C-1), 249-256.

Arthern, R. J., and R. C. A. Hindmarsh (2003), Optimal estimation of changes in the mass of ice sheets, J. Geophys. Res., 108(F1), 6007, doi:10.1029/2003JF000021.

Bamber, J. L., and R. A. Bindschadler (1997), An improved elevation dataset for climate and ice-sheet modelling: Validation with satellite imagery, Ann. Glaciol., 25, 438-444.

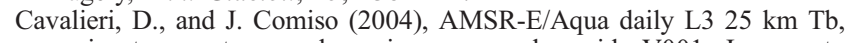
sea ice temperature, and sea ice conc. polar grids V001, January to December 2003, Natl. Snow and Ice Data Cent., Boulder, Colo.

Church, J. A., et al. (2001), Sea level change, in Climate Change: The Scientific Basis, edited by J. Houghton et al., Cambridge Univ. Press, New York.

Colbeck, S. C. (1991), The layered character of snow covers, Rev. Geophys., 29, $81-96$.

Comiso, J. C. (2000), Variability and trends in Antarctic surface temperatures from in situ and satellite infrared measurements, J. Clim., 13(10), $1674-1696$.

Genthon, C., and G. Krinner (2001), Antarctic surface mass balance and systematic biases in general circulation models, J. Geophys. Res., 106(D18), 20,653-20,664.

Giovinetto, M. B., and H. J. Zwally (2000), Spatial distribution of net surface accumulation on the Antarctic ice sheet, Ann. Glaciol., 31, $171-178$.
Goodwin, I. D. (1988), Firn core data from shallow drilling in eastern Wilkes Land, East Antarctica, ANARE Res. Not. 65, Antarctic Div., Hobart, Australia.

Huybrechts, P., D. Steinhage, F. Wilhelms, and J. L. Bamber (2000), Balance velocities and measured properties of the Antarctic ice sheet from a new compilation of gridded data for modelling, Ann. Glaciol., 30, 5260 .

Joughin, I., and S. Tulaczyk (2002), Positive mass balance of the Ross Ice Streams, West Antarctica, Science, 295(5554), 476-480.

Kitanidis, P. K. (1997), Introduction to Geostatistics: Applications to Hydrogeology, Cambridge Univ. Press, New York.

Kojima, K. (1964), Densification of snow in Antarctica, in Antarctic Snow and Ice Studies, Antarc Res. Ser, vol. 2, edited by A. P. Crary, pp. 157218, AGU, Washington, D. C.

Lehning, M., P. B. Bartelt, R. L. Brown, C. Fierz, and P. Satyawali (2002), A physical snowpack model for the Swiss Avalanche Warning Services. part III: Meteorological boundary conditions, thin layer formation and evaluation, Cold Reg. Sci. Technol., 35, 169-184.

Long, W. E. (1961), Glaciology, Byrd Station and Marie Byrd Land Traverse, 1958-1959, part XI, USNC-IGY Antarctic Glaciological Data Feild Work 1958-1959, Rep. 825-2, Ohio State Univ. Res. Found., Columbus, Ohio.

Matzler, C. (1996), Microwave permittivity of dry snow, IEEE Trans. Geosci. Remote Sens., 34, 573-581.

Pirrit, J., and G. A. Doumani (1961), Glaciology, Byrd Station and Marie Byrd Land Traverse, 1959-1960, in IGC Antarctic Glaciological Data Field Work 1959, Rep. 968-2, Ohio State Univ. Res. Found., Columbus, Ohio.

Richardson, C., E. Aarholt, S.-E. Hamran, P. Holmlund, and E. Isaksson (1997), Spatial distribution of snow in western Dronning Maud Land, East Antarctica, mapped by a ground based snow radar, J. Geophys. Res., 102(B9), 20,343-20,353.

Rignot, E., and R. H. Thomas (2002), Mass balance of polar ice sheets, Science, 297(5586), 1502-1506.

Robin, G. D. (1977), Ice cores and climatic change, Phil. Trans. R. Soc. London, Ser. A., 280, 68-143.

Stuart, A. W., and A. J. Heine (1961), Glaciology, Victoria Land Traverse, 1959-60, in IGC Antarctic Glaciological Data Field Work 1959-1960, Rep. 968-1, Ohio State Univ. Res. Found., Columbus, Ohio.

Surdyk, S., and M. Fily (1995), Results of a stratified snow emissivity model based on the wave approach: Application to the Antarctic Ice Sheet, J. Geophys. Res., 100(C5), 8837-8848.

van Lipzig, N. P. M., E. van Meijgaard, and J. Oerlemans (2002), The spatial and temporal variability of the surface mass balance in Antarctica: Results from a regional atmospheric climate model, Int. J. Climatol. 22(10), 1197-1217, doi:10.1002/joc.798.

Vaughan, D. G., and J. Russell (1997), Compilation of surface mass balance measurements in Antarctica, Internal Rep. ES4/8/1/1997/1, 56 pp., British Antarct. Surv., Cambridge, U.K.

Vaughan, D. G., J. L. Bamber, M. Giovinetto, J. Russell, and A. P. R. Cooper (1999), Reassessment of net surface mass balance in Antarctica, J. Clim., 12, $933-946$.

West, R. D., D. P. Winebrenner, L. Tsang, and H. Rott (1996), Microwave emission from density-stratified Antarctic firn at $6 \mathrm{~cm}$ wavelength, J. Glaciol., 42(140), 63-76.

Winebrenner, D. P., R. J. Arthern, and C. A. Shuman (2001), Mapping Greenland accumulation rates using observations of thermal emission at $4.5 \mathrm{~cm}$ wavelength, J. Geophys. Res., 106(D24), 33,919-33,934.

Wingham, D. J., A. L. Ridout, R. Scharroo, R. J. Arthern, and C. K. Schum (1998), Antarctic elevation change from 1992 to 1996, Science, $282,456$.

Zwally, H. J. (1977), Microwave emissivity and accumulation rate of polar firn, J. Glaciol., 18(79), 195-215.

Zwally, H. J., and M. B. Giovinetto (1995), Accumulation in Antarctica and Greenland derived from passive microwave data: A comparison with contoured compilations, Ann. Glaciol., 21, 123-130.

R. J. Arthern and D. G. Vaughan, Physical Sciences Division, British Antarctic Survey, Natural Environment Research Council, High Cross, Madingley Road, Cambridge CB3 0ET, UK. (rart@bas.ac.uk)

D. P. Winebrenner, Applied Physics Laboratory, University of Washington, Seattle, WA, USA. 